\title{
Repeatability of Measurements of Galvanic Skin Response - A Pilot Study
}

\author{
Joop Muller, Wim Pet, Ellen Pet-Reatsch", Riek Servaas, Femke Ansems, Daniel Schwander, Gary \\ Firer, Harald Lothaller and P.C. Endler
}

Interuniversity College for Health and Development, Graz / Castle of Seggau, Austria

\begin{abstract}
Objective: To scrutinize repeatability of measurements of galvanic skin response (GSR) using a complementary therapy device.

Methods: GSR diagnosis is closely related to the Chinese system of acupuncture points. These are assumed to show increased electrical conductivity that is super influenced by the (stressed or weak) state of related organs and organ systems including the vagal / simpatico system. GSR techniques apply a constant voltage. For the device used here, a "biophoton therapy device", a circuit includes a brass electrode that is held in one hand of the test person, and a style electrode for contact with his or her other hand. When the organism is exposed to external influences, skin conductivity may change within split seconds (increase or decrease). Here, it was aimed to transfer information from test substances into the circuit. Paracetamol ${ }^{\circledR}$ was dissolved in water, and both this test probe and control water were put into quartz glass vials. Following a sequence unknown to the tester, the vials were inserted into the input quartz-glass beaker of the device. A decrease in skin conductivity due to the information from Paracetamol ${ }^{\circledR}$ is assumed to be measurable at the acupuncture point "TW-1D-lefthand-pituitary gland". Repeated measurements with breaks for recovery were carried out. Three sub studies consisted of 5 unblinded and 10 blinded tests each. Depending on the sub study, one to five different tested volunteers and testers were involved. Data were analyzed blind using a two-way analysis of variance with treatment (i.e. test or control) and sub study as independent variables and the frequency of diagnosed indicator drops as the dependent variable. Furthermore, frequency of diagnosed indicator drops was compared to the frequency of non drops both for the test and the control probe in four field tables. In a random distribution, one would expect about $50 \%$ in each of the four fields.
\end{abstract}

Results: A significant main effect for treatment group was observed over all three studies $(\mathrm{p}<.001)$. The mean frequency of diagnosed indicator drops was higher for the test probe than it was for control. No main effect was found for sub study ( $>$ >.05), indicating that there were no differences between the three sub studies. In addition, no interaction effect between the variables treatment group and sub study was found ( $>>.05)$. This means that (a) the significant overall difference between treatment groups holds for each sub study in the same way and (b) the non-difference between sub studies holds both for intoxication and non-treatment. In terms of the pooled results of all three substudies the test probe was correctly detected in $77.5 \%$ of cases, with $22.5 \%$ false positive results. In reverse, the neutre control was correctly diagnosed in $68.6 \%$, with $31.4 \%$ false negative results.

Conclusion: Results suggest that information from substances can influence biological systems and that measurements of electric skin conductivity, as used for diagnostic purposes, are repeatable under standardized and blind conditions.

Keywords: Galvanic Skin Response, acupuncture points, electric skin conductivity.

\section{INTRODUCTION}

The device scrutinized in this study (a "biophoton therapy device") claims to be a non invasive diagnostic and therapeutic tool [1].

\section{DIAGNOSIS}

Galvanic skin response (GRS) diagnosis before therapy, as it is used here, does not necessarily follow the lines of western physiology. In contrast, it is closely related to the Asian (Chinese) system of acupuncture meridians and acupuncture points [2]. These acupuncture points are

*Address correspondence to this author at the Interuniversity College for Health and Development, Graz / Castle of Seggau, Austria;

Tel: 00433164238 13; E-mail: college@inter-uni.net assumed to show increased electrical conductivity [1,3-5], that is super influenced by the (stressed or weak) state of related organs and organ systems including the vagal / simpatico system [3]. Presumably Richard Croon was the first to discover the different electrical resistance values of the acupuncture points and developed the so-called Electro Neural Diagnosis [3]. In the 1960's, the technique was widely disseminated in Europe by Reinhard Voll and further developed as Electro Acupuncture by Voll [4]. Also in Japan early Electro Acupuncture methods originated (Yoshio Nakatani and Hiroshi Motoyama) [3]. This form of diagnosis is said to be particularly suitable in the case of chronic illness, the assumption being that it is capable of detecting hidden inflammations and toxins / environmental poisons within organ systems which are considered the source of illnesses [3,5]. 


\section{GALVANIC SKIN RESPONSE}

The Galvanic Skin Response (GSR) is one of several electro dermal responses. Electro dermal responses are changes in the electrical properties of a person's skin caused by an interaction between environmental events and the individuals' physical and psychological state. Human skin is a good conductor of electricity even if a weak electrical current is applied. Galvanic Skin Response techniques apply a constant voltage. The circuit normally includes a brass electrode to be hold in one hand or a plate to put the hand or bare foot on, and a style electrode. The current that flows through the skin, as the voltage is applied, can be detected and displayed [6]. When the organism is exposed to internal or external influences, this conductivity may be chronically prominent in comparison to normal, or it may change within split seconds. Chronical prominence of skin conductivity under exposure to a standard low Voltage current is the basis of clinical biophoton diagnosis. Changes within seconds or split seconds are the basis of testing materials, chemicals or information "becoming" or "unbecoming" to the tested person. In general, a "becoming" (harmonizing) information (e.g. a nutrient or a remedy) is said to lead to an increase of skin conductivity, expressed by an indicator climb, neutre information to no change and "unbecoming" (disharmonic) information to a decrease of skin conductivity, expressed by an indicator drop. By using a special low pressure measuring technique, the style electrode of the biophoton therapy device used in the studies reviewed here is reported to be distinct from older measuring electrodes and minimizes the influence of the tester and the type of skin tested [8]. It may be interesting to note that the scale showing the decrease in skin conductivity is broader in the device used here than in devices of Electro Acupuncture according to Voll.

\section{INFORMATION TRANSFER}

When the influence of becoming or unbecoming information is tested (on clients in order to find a therapeutic decision or on volunteers in the course of an experiment), the probe may be swallowed, or put directly on the bare skin, or it may be sealed in a (quartz) glass vial that is put on the skin or a mucous membrane [7]. Furthermore, the test probe may (directly or contained in a glass vial) be placed in a brass beaker (cup) that is connected to the diagnosis/therapy device. Connection may be via a closed circuit or involve one individual wire only. In the case of the biophotone therapy device scrutinized here, the individual single connection is a glass fibre cable. According to the manufacturer, glass fibre was chosen with regard to the photon nature of the information transfer. Various frequency passes may be applied, from "all pass" via " >10000 Hz", “ $>5000 \mathrm{~Hz}$, " $>2000$ etc. to "> $>100 \mathrm{~Hz}$.

\section{TREATMENT}

Treatment is based on the assumption that every biochemical reaction in organisms is preceded by electromagnetic signals, among others in the visible (VIS) or "biophoton" range, and that such photon patterns may be scanned by the device, containing one-way fibre glass cables, filters, amplifiers and elements for phase correction, and may be fed (back) to the biological system [1, 8]. Analogously, it is assumed that information from chemicals etc. may be transferred via the device.

In order to investigate possible effects of treatment with the biophoton device, pilot studies were performed previously, with promising results [9-11].

\section{RESEARCH QUESTIONS}

Based on the abovementioned assumptions, it should be possible to measure the influence of a "becoming" (harmonizing) and an "unbecoming" (disharmonizing) probe on GSR, skin conductivity. This study is organized in tree subsequent blind experiments, with three step by step research questions.

1. (" $1 V-1 R$ "): Is there a repeatedly measurable impact of a specific probe, tested versus control, on one volunteer's GSR, diagnosed by one single tester?

2. (" $5 V-1 R$ "): Is there a repeatedly measurable impact of the specific probe, tested versus control, on several volunteers' GSR, diagnosed by one single tester?

3. (" $5 V-5 R$ "): Is there a repeatedly measurable impact of the specific probe, tested versus control, on several volunteers' GSR, diagnosed by several testers?

\section{METHODS}

\section{Test Probe and Device}

The study design requires a substance that, in Galvanic Skin Response (GSR) measurement, is known to lead to a decrease of GSR. Here, the drug Paracetamol ${ }^{\circledR}$ (N-acetyl-paminophenol. N-acetyl-p-aminophenol) was used. Both in animals and in humans the substance has an analgesic and fever-reducing effect $[12,13]$. This pharmacological effect is probably caused by inhibition of an enzyme hormone synthesizing system (prostaglandin synthetase) in the brain (hypothalamus). In spite of its obvious beneficial effects in everyday clinical and over the counter healthcare, side effects $[14,15]$ and mild overdose effects were reported [16, 17] and adherents of "holistic" approaches in medicine argued that Paracetamol ${ }^{\circledR}$ on the long run use and abuse reduces the capacity of the organism for self regulation and self healing (personal communication). This led to preliminary experiments on information transfer from that substance via the "Biophoton Therapy Device" showing a decrease in GSR at the acupuncture point "TW-1D-lefthandpituitary gland" (unpublished material). Thus here, Paracetamol ${ }^{\circledR}$ is considered as an agent that, although useful in conventional medicine, uses to lead to a decrease in GSR.

$500 \mathrm{mg}$ Paracetamol ${ }^{\circledR}$ were dissolved in $1 \mathrm{ml}$ noncarbonated mineral water (test probe), and $1 \mathrm{ml}$ noncarbonated mineral water was used as the control probe. At all times, a minimum distance of $80 \mathrm{~cm}$ was maintained between the containers. Both test and control probe were put into separate quartz glass vials.

Following a sequence unknown to the tester, the vials with test and control probes were inserted into the "input" quartz-glass beaker of the "Biophoton Therapy Device J. Boswinkel" (Health Angel International AS, type Chiren 4746, Germany). 


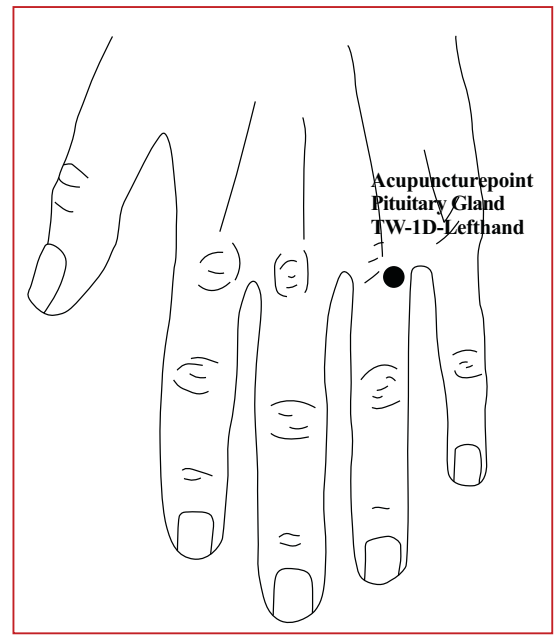

Fig. (1). Measurement point for the GSR effect of the probe. For explanation, see text.

Table 1. Pilot Phase of Study 1 (“1V-1R”). o, Measurement; t, test Probe Put into the Input Beaker; Break, Break of 5 Minutes. For Explanation see Text

1. Pilote Phase

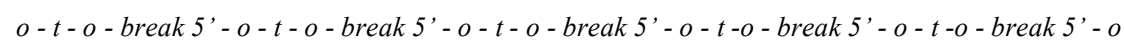

Table 2. Main Phase of Study 1 ("1V-1R"). t/c, test or Control Probe Put into the Input Beaker. For further Explanation see Legend to Table 1

1. Main Phase

$o-t / c-o-$ break 5' $-o-c-o-$ break 5' $-o-t / c-o-$ break 5' $-o-t / c-o-$ break $5^{\prime}-o-t / c-o-$ break 5' $-o$

\section{Measurement Point, Timing, Observed Parameter}

Changes in GSR due to Paracetamol ${ }^{\circledR}$ are assumed to be measurable at the acupuncture point "TW-1D-lefthandpituitary gland" (Fig. 1). One measurement was given 30 sec, and GSR values were expected to recover within $5 \mathrm{~min}$ after application of the test probe.

A brass electrode moistened with water was held in the volunteer's right hand. This hand electrode was connected to the device by an electric cable. The tester, a trained biophoton therapist (i.e. with a minimum of 3 years training which, in the Netherlands, is equivalent to a Bachelor's level), tipped the pin of the measuring style electrode onto the abovementioned acupuncture point. According to the manufacturer, this style electrode is specially designed to balance variations in pressure applied by the tester. The observed parameter was the decrease of GSR, expressed by an indicator drop on the analogue display of the device (arbitrary units) combined with an acoustic signal.

An additional person (assistant) observed / listened to the results and noted them into the protocol.

\section{Design and Participants}

All volunteers were treated with the standard "harmonizing" programme of the therapy device before the onset of the measurements. During the experiment, following a first ("warming up") measurement, the assistant put the test or control probe in the input beaker which is connected to the biophoton therapy device by a glass fibre cable. This was given 30 seconds. Then, the second measurement was performed, this was given $2 \mathrm{sec}$. Following, there was a 5 minutes break to let reset the original basic value of GSR at the acupuncture point. This was repeated for a total of 5 applications of test or control probe, 5 breaks and 11 measurements per sequence. Each part of the study consisted of 5 unblinded pilot sequences and 10 blinded main sequences. In the study design 2 (" $5 \mathrm{~V}$ $1 R$ ") the volunteers were rotating during the measurements and in the study design 3 (" $5 V-5 R$ ") both volunteers and testers were rotating. The studies took part in therapist's rooms in Ter Aar and Haarlem, The Netherlands. Volunteers ( 2 male, 3 female) were between 32 and 65 years old; testers (1 male, 4 female, JM, RS and FA, see list of authors) were between 30 and 56 years old. All volunteers and testers were in good subjective health.

1 (" $1 V-1 R$ "): Table $\mathbf{1}$ shows the protocol of one sequence of the unblinded pilot phase. 11 measurements were involved, with 5 applications of the test probe and five breaks for recovery of GSR values. One volunteer, one tester and one assistant were involved, and the sequence was applied to the volunteer 5 times subsequently.

Table 2 shows the protocol of one sequence of the blinded main phase. 11 measurements were involved, with 5 applications of the test or control probe and five breaks for recovery of GSR values. One volunteer, one tester and one assistant were involved, and the protocol was applied to the volunteer 10 times subsequently. 
Table 3. Means and Standard Deviations for all three Sub Studies. For Explanation see Text

\begin{tabular}{|c|c|c|c|}
\hline & Test & Control & p \\
\hline \hline Sub study 1 & $0.70 \pm .47$ & $0.27 \pm .45$ & 001 \\
\hline Sub study 2 & $0.83 \pm .38$ & $0.30 \pm .47$ & $<.001$ \\
\hline Sub study 3 & $0.77 \pm .43$ & $0.40 \pm .50$ & .005 \\
\hline Overall & $0.78 \pm .42$ & $0.31 \pm .47$ & $<.001$ \\
\hline
\end{tabular}

Table 4. Four Field Table on Study 1. (1V-1R). For Explanation See Text

\begin{tabular}{|c|c|c|}
\hline 1V-1R & No Drop & Drop \\
\hline \hline control: $30(100 \%)$ & $22(73 \%)$ & $8(26.7 \%)$ \\
\hline test probe: $20(100 \%)$ & $6(30 \%)$ & $14(70 \%)$ \\
\hline $\mathrm{P}<0.01$ & & \\
\hline
\end{tabular}

Table 5. Four Field Table on Study 2. (5V-1R). For Explanation See Text

\begin{tabular}{|c|c|c|}
\hline 5V-1R & No Drop & Drop \\
\hline \hline control: $20(100 \%)$ & $14(70 \%)$ & $6(30 \%)$ \\
\hline test probe: $30(100 \%)$ & $5(16.7 \%)$ & $25(83.3 \%)$ \\
\hline $\mathrm{p}<0.001$ & & \\
\hline
\end{tabular}

2. (" $5 V-1 R$ "): The protocols for the sequences of the pilot and the main phase are identical with those shown in Tables $\mathbf{1}$ and 2. Five volunteers, one tester and one assistant were involved, and the protocol was applied to each of the 5 volunteers once in the pilot phase and twice in the main phase.

3. (" $5 V-5 R$ "): The protocols for the sequences of the pilot and the main phase are identical with those shown in Tables 1 and $\mathbf{2}$. Five volunteers, 5 testers and one assistant were involved, and the protocol was applied to each of the 5 pairs of volunteers + testers once in the pilot phase and twice in the main phase.

The study design was approved by the ethics committee of the Interuniversity College.

\section{Statistical Analysis of Data}

The protocol forms were analyzed blind by the statistical department of the Interuniversity College Graz, Austria, using a two-way analysis of variance (IBM SPSS Statistics 20). Comparisons of the frequency of diagnosed indicator "drops" was performed both between the two treatment groups and across the three sub studies. Drops were encoded as " 1 " and non drops as " 0 ", and means and standard deviations were calculated. Furthermore, frequencies of diagnosed drops / non drops were compared in four field tables using Pearson's chi-square tests (IBM SPSS Statistics 20) both for the test and the control probe. These frequencies were also expressed in $\%$ of real applications of test or control substance.

\section{RESULTS}

Table 3 compares the means and standard deviations of frequencies of diagnosed drops in the control and the test probe.
The means for the test probe were significantly higher than those for the control probe, both overall and for each sub study. No significant interaction was found between the variables treatment and sub study $(\mathrm{p}=.654)$. Eta ${ }^{2}$ amounted to 0.199 for the overall comparison between groups. There were no differences between sub studies, regardless of whether treatments were considered together $(p=.501)$ or separately $(\mathrm{p}=.581$ for test probe, $\mathrm{p}=.581$ for control probe). $\mathrm{Eta}^{2}$ amounted to 0.010 for the overall comparison between the sub studies.

Frequencies of diagnoses were also expressed in \% of real applications of control and of test substances (Table 4). In a random distribution, one would expect about $50 \%$ in each of the four fields. As can be seen, $73 \%$ of control applications led to "no drop" diagnoses, whereas $70 \%$ of test probe applications led to "drop" diagnoses. In other words, when one volunteer and one tester were involved, the disharmonizing test probe was correctly detected in $70 \%$ of the cases, with $30 \%$ false positive results. In reverse, the neutre control was correctly diagnosed in $73 \%$, with 26.7 false negative results.

When 5 volunteers and one tester were involved (Table 5), the disharmonizing test probe was correctly dedected in $70 \%$ of the cases, with $30 \%$ false positive results. In reverse, the neutre control was correctly diagnosed in $83.3 \%$, with 16.7 false negative results.

When non drops were coded "0" and drops " 1 ", mean \pm S.D. was $0.30 \pm 0.47$ for control and $0.83 \pm 0.38$ for the test probe.

When 5 volunteers and 5 testers were involved (Table 6), the disharmonizing test probe was correctly dedected in $76.7 \%$ of the cases, with $23.3 \%$ false positive results. In reverse, the neutre control was correctly diagnosed in $60 \%$, 
Table 6. Four Field Table on Study 3. (5V-5R). For Explanation See Text

\begin{tabular}{|c|c|c|}
\hline 5V-5R & No Drop & Drop \\
\hline \hline control: $20(100 \%)$ & $12(60 \%)$ & $23(40 \%)$ \\
\hline test probe: $30(100 \%)$ & $7(23.3 \%)$ & \\
\hline $\mathrm{p}<0.05$ & & \\
\hline
\end{tabular}

Table 7. Four Field Table on Pooled Results from Studies 1.-3. For Explanation See Text

\begin{tabular}{|c|c|c|}
\hline Pooled Results 1.-3. & No Drop & Drop \\
\hline \hline control: $70(100 \%)$ & $48(68.6 \%)$ & $22(31.4 \%)$ \\
\hline test probe: $80(100 \%)$ & $18(22.5 \%)$ & $62(77.5 \%)$ \\
\hline$p<0.001$ & & \\
\hline
\end{tabular}

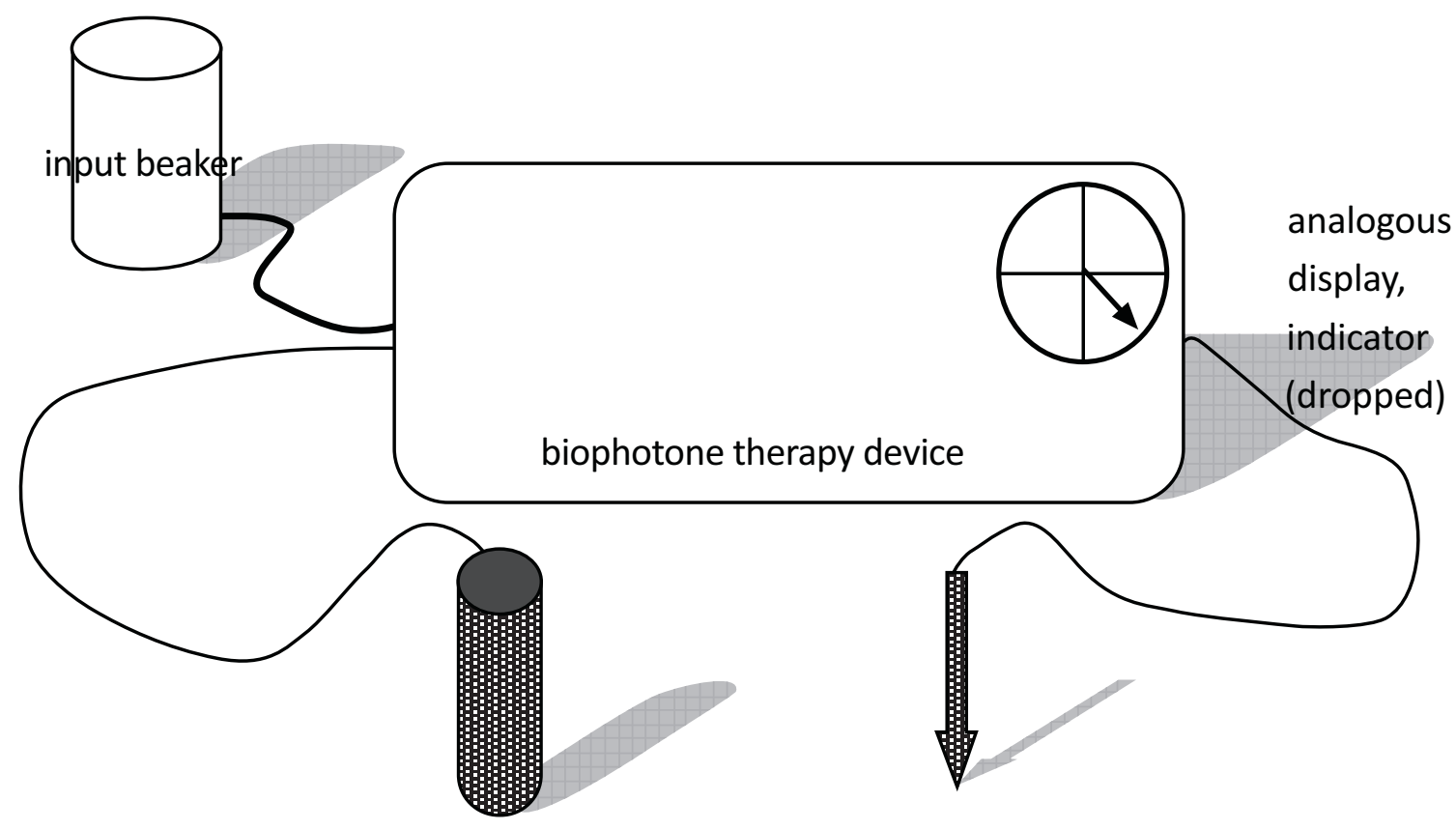

Fig. (2). Experimental setup. For explanation, see text.

with $40 \%$ false negative results. Findings are statistically significant $(\mathrm{p}<0.05)$.

When non drops were coded " 0 " and drops " 1 ", mean \pm S.D. was $0.40 \pm 0.50$ for control and $0.77 \pm 0.43$ for the test probe.

When the results of all studies 1.-3. were pooled (Table 7 and Fig. 2), the disharmonizing test probe was correctly detected in $77.5 \%$ of the cases, with $22.5 \%$ false positive results. In reverse, the neutre control was correctly diagnosed in $68.6 \%$, with $31.4 \%$ false negative results. Findings are statistically significant $(\mathrm{p}<0.01)$.

When non drops were coded " 0 " and drops " 1 ", mean \pm S.D. was $0.31 \pm 0.47$ for control and $0.78 \pm 0.42$ for the test probe.

\section{DISCUSSION}

The objective of the study was to scrutinize repeatability of measurements of galvanic skin response (GSR) using a "biophoton device".
When one volunteer and one tester were involved, information from a "disharmonizing" test probe was correctly detected in $70 \%$ of the cases, with $30 \%$ false positive results. In reverse, control was correctly diagnosed in $73 \%$, with 26.7 false negative results. Findings are statistically significant $(\mathrm{p}<0.01)$. When 5 volunteers and one tester were involved, the test probe was correctly detected in $70 \%$ of the cases and control in $83.3 \%(\mathrm{p}<0.01)$. When 5 volunteers and 5 testers were involved, the test probe was correctly dedected in $76.7 \%$ and control in $60 \%$ (p $<0.05)$. When the results of all 3 studies were pooled, the test probe was correctly detected in $77.5 \%$ of the cases, with $22.5 \%$ false positive results. In reverse, the neutre control was correctly diagnosed in $68.6 \%$, with $31.4 \%$ false negative results $(\mathrm{p}<0.01)$.

While this paper was in preparation for print, a repetition of study 2 (above) involving one tester and 23 volunteers was performed by GF and DS at the School for Natural Medicines in Zurich, Switzerland (unpublished material). Their experimental setup was in principle the same 


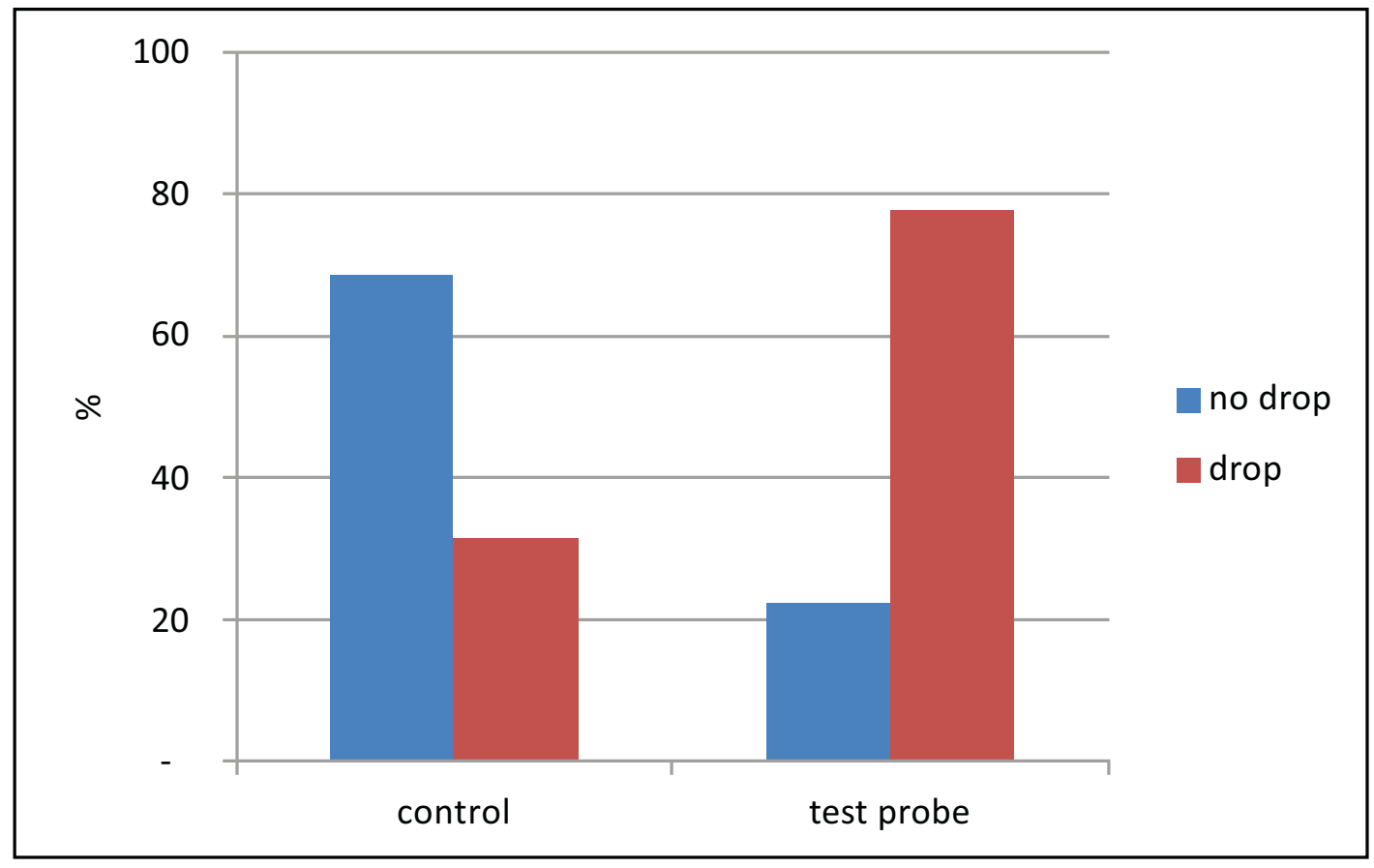

Fig. (3). Pooled results from studies 1.-3. Ordinate $=$ frequencies of diagnosed drops / non drops expressed in percent of real applications of control and of test substance. For further explanation see text.

Table 8. Four Field Table Showing the Results of the Repetition Study. For Explanation See Text

\begin{tabular}{|c|c|c|}
\hline Rep. 23V-1R & no drop & Drop \\
\hline \hline control: $230(100 \%)$ & $167(72.6 \%)$ & $63(27.4 \%)$ \\
\hline test probe: $230(100 \%)$ & $140(60.9 \%)$ & $90(39.1 \%)$ \\
\hline$p<0.01$ & & \\
\hline
\end{tabular}

as reported here, especially regarding the use of a biophoton therapy device, Paracetamol ${ }^{\mathbb{B}}$ and acupuncture point "TW1D-lefthand-pituitary gland". However, there were also methodological differences: participants were not treated with the standard "harmonizing" programme before the start of measurements; the number of measurements per participant was higher (10 instead of 5); all participants were present at the same time (albeit with those recovering or waiting between measurements in a separate room), as opposed to study 2, where participants entered the area at different times; and they had to move physically and wash their hands between sets of measurements. Furthermore, the repeat study involved more test substances and one more measuring point than study 2 , (where only one test substance was tested against control and one point was measured for GRS).

In the repeat study (Table 8, Fig. 3), the disharmonizing test probe $\left(\right.$ Paracetamol $^{\circledR}$ ) was correctly detected in $39.1 \%$ of cases, leaving $60.9 \%$ false positive results. In the reverse experiment, the neutre control was correctly diagnosed in $72.6 \%$, with $27.4 \%$ false negative results. Although the differences were less marked than in the studies 1-3, they were still statistically significant $(\mathrm{p}<0.01)$.

When non drops were coded " 0 " and drops " 1 ", mean \pm S.D. was $0.27 \pm 0.45$ for control and $0.39 \pm 0.49$ for the test probe.
The results seem to confirm that information from biophoton therapy devices can influence biological systems and that measurements of electric skin conductivity as they are used for diagnostic purposes are repeatable under double blind conditions. Further research on the model presented here is ongoing using different test substances. Further studies may investigate the influence of the methodological differences between the original and the repeat study described above. Furthermore, independent methods (such as heart rate variability) should be used to monitor effects of information transferred via the biophoton device in order to further validate the use of skin conductivity measurements as a diagnostic tool.

Results are in line with a study performed in the 1980ies $[7,18]$, using the medicine test according to Voll. A phial containing a homeopathic remedy was either placed in close proximity to a patient, or was included in a testing circuit. Electric conductivity of the skin and its changes were measured. The authors, R. van Wijk and F.A.C. Wiegant, wrote: "In both cases, the remedy is not in direct contact with the skin and the phial seems to be permeable to the 'information' which characterizes the remedy. Different series of experiments showed a significant deviation between an agitated dilution of sulphur and placebo" [18, p. 81].

Information transfer from a drug sealed in a glass vial via an electronic circuit was also described in immunological $[19,20]$ and zoological experiments $[21,22]$. Recent 
biochemical assumptions may shed new light on the issue of information transfer from molecules [23].

\section{CONCLUSION}

Results suggest that information from substances can influence biological systems and that measurements of electric skin conductivity, as used for diagnostic purposes, are repeatable under standardized and blind conditions.

\section{DISCLOSURE STATEMENT}

No competing financial interests exist.

\section{CONFLICT OF INTEREST}

The authors confirm that this article content has no conflicts of interest.

\section{ACKNOWLEDGEMENT}

Declared none.

\section{REFERENCES}

[1] Boswinkel J. Energetic anatomy, physiology and pathology. Drammen: Edition Health Angel Academy 2008.

[2] Deadman P, Al-Khafaji M, Baker K. A manual of acupuncture. China: Journal of Chinese Medicine Publications 2007.

[3] Bischof M. Das Licht in unseren Zellen. Frankfurt: Zweitausendeins Verlag 2002.

[4] Voll R. The phenomenon of medicine testing in electroacupuncture according to Voll. Am J Acupunct 1980; 8(2): 97-104.

[5] Kramer F. Lehrbuch der Elektroakupunktur. Heidelberg: HaugVerlag 1976.

[6] Fuller GD. Biofeedback methods \& procedures in clinical practice. San Francisco: Biofeedback Press 1977.

[7] van Wijk R, Wiegant FAC. Homeopathic remedies and pressureinduced changes in the galvanic resistance of the skin. Alkmaar: VSM Geneesmiddelen 1989.

[8] Boswinkel J, Kramer F. Manual chiren biontology. Drammen: Edition Health Angel Academy 2007.
[9] Vastenburg D, Pet S. Morphology of isolated rat cortical neurons and information transferred via a "biophoton device" - a pilot study. Explore 2012; 21(1): 7-12.

[10] Vastenburg D, Pet S. A follow-up study on the morphology of isolated rat cortical neurons and information transferred via a "biophoton device". J Altern Med Res 2012; 4(2): 1-8.

[11] Pet-Reatsch E, Pet W. Chronic health complaints in children and adolescents and "biophoton therapy" - a pilot study. Explore 2011; 20(6): 29-34

[12] Bohn - Stafleu - van Loghum. Merck Manual Medisch Handboek $2^{\text {nd }}$ ed. Berlin: Springer 2005.

[13] Wolffers I, Medicijnen 2008-2009. Uitgeverij Contact, Amsterdam/Antwerpen 2007.

[14] Lee WM. Acetaminophen and the U.S. acute liver failure study group: lowering the risks of hepatic failure. Hepatology 2004; 40: 6-10.

[15] Shaheen S, Sterne JAC, Songhurst CE, Burney PGJ. Frequent paracetamol use and asthma in adults. Thorax 2000; 55: 266-70.

[16] Hameleers-Snijders P. Hogeveen M, Smeitink JAM, Kramers C, Draaisma JMT. Risk of acute hepatic insufficiency in children due to chronic accidental overdose of paracetamol (acetaminophen). Ned. Tijdschr Geneeskd 2007; 151: 897.

[17] Watkins PB, Kaplowitz N, Slattery JT, et al. Aminotransferase elevations in healthy adults receiving 4 grams of acetaminophen daily - A randomized controlled trial. J Am Med Assoc 2006; 296: 87.

[18] van Wijk R, Wiegant FAC. Physiological effects of homeopathic medicines in closed phials; a critical evaluation. In: Endler PC, Schulte J, Eds. Ultra High Dilution. Physiology and Physics. Dortrecht: Kluwer 1994.

[19] Aissa J, Litime MH, Attis E, Benveniste J. Molecular signalling at high dilution or by means of electronic circuitry. J Immunol 1993; 150: A146.

[20] Benveniste J, Aissa J, Litime MH, Tsaegaca GT, Thomas Y. Transfer of the molecular signal by electronic amplification. FASEB J 1994; 8: A398.

[21] Citro M, Endler PC, Pongratz W, Vinattieri C, Smith CW, Schulte J. Hormone effects by electronic transmission. FASEB J 1995; 9 : A12025.

[22] Endler PC, Citro M, Pongratz W, Smith CW, Vinattieri C, Senekowitsch F. Übertragung von Molekül-Information mittels Bioresonanz-Gerät im Amphibienversuch. Erfahrungsheilkunde 1995; 3: 186-92.

[23] Enserink M. Newsmaker Interview: Luc Montagnier. Science 2010; 330: 1732 .

(C) Muller et al.; Licensee Bentham Open.

This is an open access article licensed under the terms of the Creative Commons Attribution Non-Commercial License (http://creativecommons.org/licenses/by$\mathrm{nc} / 3.0 /$ ), which permits unrestricted, non-commercial use, distribution and reproduction in any medium, provided the work is properly cited. 